Revue d'histoire de l'Amérique française

REYUE D.HISTOIRE DE L'AMÉRIQUE FRANÇAISE

\title{
La « république canadienne » de 1838
}

\section{Victor Morin}

Volume 2, numéro 4, mars 1949

URI : https://id.erudit.org/iderudit/801498ar

DOI : https://doi.org/10.7202/801498ar

Aller au sommaire du numéro

Éditeur(s)

Institut d'histoire de l'Amérique française

ISSN

0035-2357 (imprimé)

1492-1383 (numérique)

Découvrir la revue

Citer cet article

Morin, V. (1949). La « république canadienne » de 1838. Revue d'histoire de l'Amérique française, 2(4), 483-512. https://doi.org/10.7202/801498ar d'utilisation que vous pouvez consulter en ligne.

https://apropos.erudit.org/fr/usagers/politique-dutilisation/ 


\section{LA “RÉPUBLIQUE CANADIENNE” DE 1838}

Les insurrections canadiennes de 1837 et de 1838, de même que les rôles des principaux acteurs de ce drame politique, ont été diversement appréciés. On connaît généralement la genèse et les grandes lignes de ce soulèvement populaire qui a déclenché la conquête de nos libertés constitutionnelles; mais ceux qui n'ont pas étudié particulièrement l'histoire de ces luttes, en ignorent encore certains aspects qu'il nous semble intéressant de faire revivre à un siècle de distance et que le recul du temps nous permettra conséquemment d'apprécier avec plus d'impartialité.

Aussi le centenaire de la reconnaissance parlementaire d'un gouvernement responsable au peuple du Canada, que nous célébrons en cette année 1948, nous a-t-il paru présenter une occasion logique d'en évoquer les prodromes sanglants. Les grandes scènes en ont été fréquemment tracées par nos écrivains et nos orateurs; aussi nous bornerons-nous à en signaler certains détails moins connus: ceux qui ont gravité autour de la "République canadienne" de 1838, laissant aux auteurs de grande envergure le soin de commenter les aspects plus saisissants de cet ensemble.

La mise à jour de certains documents et pièces inconnus, ou difficilement accessibles à nos devanciers, nous permettra en outre de juger avec meilleure connaissance de cause certains événements qui nous semblaient obscurs ou qui nous ont été présentés de façon contradictoire par quelques historiens esclaves de leurs préférences ou de leurs animosités. Les collections des journaux de l'époque, recueillis par Philéas Gagnon et maintenant accessibles au public dans la bibliothèque de Montréal; la publication des papiers de Ludger Duvernay et de la relation historique du Dr Henri Brien dans le Canadian Antiquarian de la Société d'Archéologie de Montréal; les manuscrits des Archives nationales d'Ottawa et les papiers de JeanJoseph Girouard déposés aux Archives provinciales de Québec; la 
correspondance de l'Ambassade française aux Etats-Unis publice dans la revue Nova Francia; le journal intime d'Amédée Papineau dont copie se trouve à la bibliothèque Saint-Sulpice; les notes laissées par Aegidius Fauteux pour la publication de son Dictionnaire des Patriotes de 1837-1838; tous ces documents et d'au tres de même nature nous permettront, espérons-le, de fournir quelques nouveaux éclaircissements à ceux qui prennent un intérêt particulier à cette période mouvementée de notre histoire.

Après un succès passager à Saint-Denis, l'insurrection des $\mathrm{Pa}$ triotes révoltés contre une oligarchie systématiquement combinée venait d'être noyée dans le sang à Saint-Charles et à Saint-Eustache. Les quelque centaines d'enthousiastes qui avaient cru possible de résister avec des fourches et des bâtons pointus aux troupes bien armées de Colborne, avaient payé de leur vie le crime de chercher à conquérir par la force une liberté qui leur était garantie par les traités mais neutralisée par la bureaucratie. Les chefs avaient péri les armes a la main ou cherché refuge aux Etats-Unis et leurs têtes avaient été mises à prix; ils y avaient été suivis par de nombreux partisans qui surveillaient la frontière canadienne cherchant l'occasion de prendre leur revanche. Ceux qui n'avaient pu traverser la frontière avaient été mis en prison pour y attendre leur comparution en Cour martiale; d'autres personnages en vue, bien que n'ayant pris aucune part au soulèvement, avaient également été arrêtés puis relâchés après quelques jours d'emprisonnement sans avoir pu obtenir de procès.

La révolte était matée, mais les causes qui l'avaient déterminée continuaient d'exister: la violation du pacte constitutionnel, l'appropriation autoritaire des deniers publics sans le contrôle de la chambre d'assemblée, en un mot l'abus du pouvoir au mépris des droits du peuple continuaient de s'afficher avec une insolence rendue plus grande encore par l'écrasement des rebelles.

Réfugiés dans un pays qui avait conquis ses libertés dans des conditions quasi identiques un demi-siècle auparavant, les meneurs du mouvement populaire crurent possible d'intéresser la République américaine à leur cause; ills oubliaient que les gouvernements n'entrent pas en guerre pour le triomphe d'un principe, quelque méritoire qu'il 
soit, à moins d'y avoir un intérêt direct; et leurs démarches auprès des autorités de ce pays furent stériles. Quelques groupes isolés de citoyens américains manifestèrent cependant leur sympathie aux révoltés du Haut et du Bas-Canada par des actes en leur offrant spontanément leur concours dans l'organisation d'une reprise d'hostilités; mais, là encore, les autorités intervinrent auprès de leurs administrés pour faire respecter la neutralité de deux nations amies.

Cependant, l'agitation continuait sourdement chez les groupes canadiens échelonnés près de la frontière. On tenait des assemblées auxquelles les chefs, assagis par l'expérience, conseillaient la prudence, tandis que les exaltés activaient la flamme d'un nouvel incendie. Louis-Joseph Papineau restait toujours l'idole en qui les Patriotes avaient placé leur confiance. Il avait eu de fréquentes conférences avec le gouverneur de New York à Albany ainsi qu'avec divers financiers, et il se faisait fort de négocier un emprunt de $\$ 200,000$. pour financer une invasion que les généraux Scott et Wool lui avaient offert de conduire au Canada avec des troupes aguerries, mais il hésitait à en assumer la responsabilité devant l'insuffisance des préparatifs. Nous verrons bientôt que ces mêmes généraux lui en gardèrent rancune et qu'ils n'hésitèrent pas à se rendre à l'appel du gouverneur Marcy pour faire respecter la neutralité des Etats-Unis dès que les révoltés se montrèrent les armes à la main.

Une assemblée consultative des chefs du mouvement eut lieu à Middleburg, le ler janvier 1838, pour établir le problème des revendications et préparer le plan d'action. Papineau s'y rendit avec O'Callaghan, Rodier, Robert Nelson, Côté, Mailhot, Bouthillier, Davignon et nombre d'autres, mais on ne put s'entendre sur tous les points; il y eut même de vives altercations entre les tenants de l'agression immédiate: Nelson, Côté et Rodier d'une part, contre les temporisateurs: O'Callaghan et Papineau d'autre part, si bien que ceux-ci décidèrent de se tenir à l'écart des préparatifs. ${ }^{1}$

Parmi les plus chauds partisans de la revanche armée se trouvait Robert Nelson, frère cadet de celui qui avait déclaré à l'assemblée des Six Comtés, l'année précédente, que le temps était arrivé pour les Canadiens de "fondre leurs cuillers de plomb pour en faire des balles"

1. On reprochait à Papineau de s'opposer à l'abolition de la tenure seigneuriale par in térêt personnel, vu que sa famille était propriétaire d'une seigneurie. 
et qui avait ainsi déclenché l'insurrection. Praticien de haute renommée, orateur convaincu, Nelson enthousiasma ses auditeurs au point de les déterminer à déclarer la rupture du lien colonial, l'envahissement du Canada pour se joîndre à l'armée de 5,000 hommes qui les attendaient, disait-il, à Napierville et l'établissement de la REPUBLIQUE DU BAS-CANADA.

Il n'avait pris aucune part au soulèvement de 1837, mais il avait tout de même été arrêté comme suspect le 24 novembre précédent à cause de son étroite parenté avec Wolfred Nelson; libéré sous caution le lendemain, par suite d'irrégularités dans le mandat, on dit qu'il écrivit sur le mur de sa cellule: "Le Gouvernement anglais se souviendra de Robt. Nelson" 2; il tint parole. I! devint bientôt au nombre des plus actifs partisans de l'invasion armée.

Devant l'inaction de Papineau, les réfugiés de Swanton signent une adresse offrant à Robert Nelson de conduire le mouvement insurrectionnel. Celui-ci se met aussitôt en rapport avec les chefs de groupes des principaux centres du Bas-Canada, tandis que William-Lyon Mackenzie préparait, du côté de Buffalo, l'invasion du Haut-Canada sur divers points de la frontière américaine.

Le premier soin de Nelson fut de rédiger une Déclaration d'Indépendance avec proclamation d'une République du Bas-Canada; en voici le texte que nous croyons utile de rapporter ici, vu qu'on le trouve rarement dans nos traités d'histoire et que cette déclaration résume les griefs du peuple ainsi que les réformes dont il réclamait l'adoption.

\section{DÉCLARATION D'INDÊPENDANCE}

$V u$ que le pacte solennel fait entre le peuple du Haut et du Bas Canada, enregistré dans le livre des statuts du Royaume Uni de la Grande Bretagne et d'Irlande, le 31è chapitre des Actes passé dans la 31è année du règne du Roi Georges III, a été continuellement violé par le Gouvernement Britannique.

$V u$ que le même Gouvernement a foulé aux pieds et usurpé nos droits, qu'il a méprisé et fermé l'oreille à nos adresses, requêtes, protêts et remonstrations contre son intervention inconstitutionnelle et injuste dans nos affaires; qu'il a disposé de nos revenus sans le consentement constitutionnel de la Législature locale, pillé notre trésor colonial, ordonné l'arrestation de plusieurs de nos concitoyens et leur mise aux chaines; jeté au milieu de nos campagnes des

2. Cf. Journal d'un Fils de la Liberté réfugié aux Etats-Unis par suite de l'insurrection canadienne en 1837 par L.-J. Amédée Papineau, en 6 cahiers manuscrits. 
Armées de mercenaires qui y ont semé l'alarme, l'effroi et la consternation; que la même soldatesque a rougi notre sol du sang d'un nombre considérable de nos compatriotes, brûlé nos villages, profané nos temples, établi dans toute l'étendue du pays le règne le plus atroce de la terreur;

Et vu que nous ne pouvons plus souffrir ces violations réitérées de nos droits les plus sacrés et supporter patiemment les outrages et les cruautés multipliées et récentes du Gouvernement du Bas Canada,

Nous, au nom du Peuple du Bas Canada, adorant les décrets de la Divine Providence, qui nous permet de renverser un Gouvernement qui à méconnu l'objet et l'intention pour lequel il était créé, et de faire choix de la forme de Gouvernement la plus propre à établir la justice, assurer la tranquillité domestique, pourvoir à la défense commune, promouvoir le bien général et garantir à nous et à notre postérité les bienfaits de la Liberté civile et religieuse.

\section{DÉCLARONS SOLENNELLEMENT}

1. - Qu'à compter de ce jour, le Peuple du Bas Canada est absous de toute allégeance à la Grande Bretagne, et que toute connexion politique entre cette puissance et le Bas Canada Cesse dès ce jour.

2. - Que le Bas Canada doit prendre la forme d'un gouvernement Républicain et se déclare maintenant, de fait, République.

3. - Que sous le Gouvernement libre du Bas Canada, tous les citoyens auront les mềmies droits; les Sauvages cesseront d'être sujets à aucune disqualification civile quelconque, et jouiront des mêmes droits que les autres citoyens de l'État du Bas-Canada.

4. -Que toute union entre l'Église et l'État est déclarée abolie, et toute personne a le droit d'exercer librement la religion et la croyance que lui dicte sa conscience.

5. - Que la Tenure Féodale ou Seigneuriale est, de fait, abolie, comme si elle n'ê̂t jamais existé dans ce pays.

6. - Que toute personne qui portera les armes ou fournira des moyens d'existence au Peuple Canadien dans sa lutte d'émancipation, est déchargée de toutes dettes ou obligations, réelles ou supposées, envers les Seigneurs, pour arrérages en vertu de Droits Seigneuriaux ci-devant existants.

7. - Que le Douaire Coutumier est, à l'avenir, entièrement aboli et prohibé.

8. - Que l'emprisonnement pour dettes n'existera plus sauf les cas de fraude évidente que l'on spécifiera dans un acte de la Législature du Bas Canada à cet effet.

9. - Que la peine de mort ne sera prononcée que dans le cas de meurtre seulement et pas autrement.

10. -Que toute Hypothèque sur Biens-Fonds devra être spéciale et, pour être valide, devra être enregistrée dans les Bureaux créés à cet effet par un acte de la Législature du Bas-Canada.

11. - Qu'il y aura liberté pleine et entière de la Presse dans toutes les matières et affaires publiques. 
12. - Que le Procès par Jury est garanti au Peuple de l'État dans son étendue la plus libérale dans les procès criminels et dans les affaires civiles au montant d'une certaine somme à être déterminée par la Législature du Bas Canada.

13. - Que, comme une nécessité et un devoir du Gouvernement envers le Peuple, l'Education publique et générale sera mise en opération et encouragée d'une manière spéciale, aussitôt que les circonstances pourront le permettre.

14. - Que pour assurer la franchise et la liberté élective, toute élection se fera par le moyen du Ballot.

15. -Qu'aussitót que les circonstances pourront le permettre, le Peuple choisira des Délégués suivant la division actuelle du pays dans les Villes, Bourgs et Comtés, qui constitueront une Convention, ou Corps Législatif, afin de baser et d'établir une Constitution, selon les besoins du Pays, et conformément aux dispositions de cette Déclaration, sujette à être modifiée suivant la volonté du Peuple.

16. - Que toute personne mâle au-dessus de l'âge de vingt et un ans aura le droit de voter ainsi que pourvu ci-dessus, pour l'élection des Délégués susnommés.

17. - Que toutes les Terres dites de la Couronne ainsi que celles appelées Réserves du Clergé et celles qui sont nominalement en possession d'une certaine Compagnie de spéculateurs en Angleterre, appelée "Compagnie des Terres de l'Amérique Britannique du Nord" deviennent, de plein droit, la propriëté de l'État du C'anada, sauf telles portions des dites terres qui peuvent être en possession de cultivateurs qui les tiennent de bonne foi, pour lesquelles nous garantissons des titres en vertu d'une loi qui sera passée afin de légaliser la possession de tels lots de terre situés dans les Townships, qui sont maintenant en culture.

18. -Qu'on se servira des langues Firansaise et Anglaise dans toute matière publique.

Et pour le support de Cette Déclaration et le succès de la cause Patriotique que nous soutenons, Nous, confiants en la protection du Tout-Puissant et la justice de notre ligne de conduite, engageons, par ces présentes, mutuellement et solennellement les uns envers les autres, notre vie, nos fortunes et notre honneur le plus sacré.

Par ordre du Gouvernement Provisoire Robert Nelson, Président ${ }^{3}$

Cette Déclaration d'Indépendance n'est pas datée, mais elle fut adressée aux journaux, dans les derniers jours de février 1838, pour fins de publication.

Dans le but d'obtenir le concours de ceux qui seraient disposés à aider l'invasion armée du Canada, ainsi que de se concilier la neutralité

3. Dans le même temps, William-Lyon Mackenzie proclamait la République du Haut-Canada. 
des autres, Robert Nelson, assumant cette fois le titre de Commandant en Chef de l'Armée des Patriotes, émettait aussitôt une autre Proclamation à cet effet dont le style se ressent de la précipitation évidente des événements. Elle est rédigée dans les termes suivants:

\section{PROCLAMATION \\ NO. 2 \\ PEUPLE DU CANADA}

Nous avons été opprimés par la main d'un pouvoir transatlantique et chaties injustement et cruellement par la verge d'un mauvais gouvernement non interrompu pour nombre d'années - si longtemps que les mesures tyranniques sont devenues à leur comble. Nous avons incessamment essayé, mais en vain, à remodeler le gouvernement, à abolir les mauvaises lois - et en établir qui auraient pu faire sortir nos institutions de leur ancien etat de vasselage et de les élever au niveau de celles qui caractérisent les gouvernements récents du 19ème siècle. Nous sommes maintenant contraints par la force de la Tyrannie et en dépit [ de] nos sentiments, d'appeler à la force des armes dans la vue d'acquérir et de nous procurer les droits qui sont dus à un peuple libre et digne. Nous ne déposerons les armes que lorsque nous aurons procuré à notre pays l'avantage d'un gouvernement patriote et responsable.

$A$ toutes personnes qui nous aideront dans ces entreprises patriotiques, nous leur tendons une main fraternelle et amie. Et à tous ceux qui persisteront dans le cours aveugle, entêté, sanguinaire, incendiaire et de pillage qui, à notre chagrin et aux souffrances de notre peuple, de nos femmes et de nos enfants, a caractérisé d'une manière si disgracieuse la carrière étourdie de sir John Colborne, commandant des forces britanniques, et ses adhérens, nous emploierons pour notre propre défense et la justice commune de notre peuple et de notre cause, la voie de la revanche que leur exemple nous a donné pour précédent.

Mais comme il y a beaucoup de personnes qui se repentent de leur conduite et du vandalisme de leurs associés - état de choses qui nous a poussés à la guerre; et comme nos sentiments d'humanité, de justice et d'honneur ont été coulés dans un meilleur moule que ceux de nos oppresseurs, nous ne pouvons réconcilier avec nos principes ou avec la morale qui, ailleurs que dans le gouvernement anglais du Canada, distingue l'áge où nous vivons d'exercer à leur égard leur sauvage exemple.

Nous, par conséquent, promettons solennellement d'accorder sécurité et protection, et dans la personne et dans la propriété, à toutes et telles personnes qui mettront bas les armes et qui autrement cesseront de nous opprimerpromesse que notre caractère et les habitudes connues, morales et paisibles de notre population garantissent suffisamment. Ni ne mettrons-nous bas les 
armes, jusqu'à ce que nous ayons effectué et atteint l'objet de notre première proclamation.

Par ordre du gouvernement provisoire de l'État du Bas-Canada.

Robert Nelson

Commandant-en-chef de l'armée patriote.

A peine ces appels étaient-ils lancés qu'un groupe de quelques centaines de Patriotes, conduits par le commandant Nelson et l'enthousiaste Dr C.-H.-O. Côté (celui-là même qui faisait prêter le serment libertaire à l'assemblée des Six Comtés l'année précédente), s'étant procuré deux cent cinquante fusils à l'arsenal d'Elizabethtown, se mettaient en marche en vue d'envahir le Canada. Partis d'Alburg, dans l'Etat du Vermont, le 28 février 1838, ils avaient à peine parcouru un mille en territoire canadien qu'ils furent attaqués par deux corps de troupes accourus de Missisquoi et de Henryville, sous les ordres des colonels Ward et Booth, et refoulés à la frontière américaine où le général Wool, envoyé à leur poursuite par le gouverneur Marcy, leur fit mettre bas les armes et s'en empara. Nelson et Côté furent emprisonnés aux Etats-Unis pour atteinte à la neutralité d'un pays où ils avaient reçu asile, mais acquittés par un jury sympathique.

Instruits par cet échec, les chefs du mouvement se rendirent compte que l'enthousiasme et la détermination ne suffisent pas à assurer le succès d'une cause. Comme ils étaient sincères et tenaces dans leurs revendications, ils mirent sur pied un plan d'organisation militaire entourée du plus grand secret, dont les ramifications devaient s'étendre aux Etats-Unis, depuis le Maine jusqu'au Michigan,et dans tous les comtés du Canada. C'était l'Association des Frères Chasseurs dont les adeptes juraient de garder les secrets qui leur seraient confiés "sous peine de voir leurs propriétés détruites et d'avoir le cou coupé jusqu'à l'os". Nombre de braves et pacifiques habitants du Bas-Canada, épouvantés de ces menaces, prêtèrent le serment requis d'eux, mais sans enthousiasme, à seule fin de protéger leurs vies et leurs biens.

Comme les défaites lamentables du passé étaient surtout attribuables à l'ignorance de la stratégie militaire, cette Association fut constituée, dans ses cadres restreints, sur le modèle d'une armée; elle obéissait à la direction d'un commandant en chef qui avait le titre de Grand Aigle et dont les fonctions correspondaient à celles d'un général 
de division; il avait sous ses ordres les Aigles de chaque district qui remplissaient le rôle de colonels et avaient charge d'organiser une compagnie; ils choisissaient à cet effet deux officiers nommés Castors dont le rang équivalait à celui de capitaine et chacun d'eux nommait à son tour cinq caporaux désignés sous le nom de Raquettes. Enfin chaque Raquette commandait à neuf hommes qui étaient les Chasseurs proprement dit. Les initiés se reconnaissaient entre eux par des signes et mots de passe ${ }^{4}$ et chaque ville ou paroisse compta bientôt une loge de Chasseurs.

Pour prélever les fonds nécessaires à la mise en marche de l'insurrection, des souscriptions furent clandestinement recueillies chez les amis du mouvement au Canada. On alla même jusqu'à vouloir s'emparer des caisses de Fabriques paroissiales et cette piraterie semblait d'autant plus légitime aux yeux des chefs que les autorités religieuses avaient condamné la rébellion sous peine de refus des sacrements de l'Église.

Cette organisation, mise en marche au printemps de 1838, se développa jusqu'au samedi 3 novembre suivant, date fixée pour le soulèvement général du Bas-Canada, avec centre de ralliement à Napierville où les chefs Nelson, Côté et Gagnon arrivèrent dans la matinée, accompagnés de deux officiers français du nom de Hindenlang et Touvrey et de deux officiers polonais nommés Oklomski et Szesdrakowski sur qui ils comptaient pour diriger les opérations militaires. Environ 2,000 hommes s'étaient rendus à cet endroit, à l'appel des chefs, pour constituer le gros de l'armée.

Le plan général d'invasion, combiné avec W.L. Mackenzie et les autres chefs du Haut-Canada, comportait une offensive sur toute la frontière américaine, à partir du Maine jusqu'au Michigan, qui devait précéder de cinq jours des attaques simultanées contre Beauharnois, Châteauguay, Laprairie, Saint-Jean, Chambly, Boucherville et Sorel

4. Antoine Coupal, cultivateur de l'Acadie, nous dit, dans une déposition faite à Monitréal, le 23 novembre 1838, en quoi consistaient ces signes et mots de passe. Il avait reçu ordre de Julien Gagnon, de François Nicolas et du Dr Cóté, de conduire les gens de sa Côte (le ruisseau des Iroquois) à la Pointe à la Mule et d'aller sommer le marguillier Louis Béchard de lui remettre l'argent de la Fabrique, ce que celui-ci lui refusa. Voici comment il s'exprime à ce sujet: "Je lui fis alors les signes en me mettant le pouce gauche dans le nez et passant le doigt dessus, disant en même temps: Un beau temps aujourd'hui et ne m'ayant point répondu, je jugeai alors que cet homme nous était dangereux; je le liai et me mis en chemin pour le conduire prisonnier au camp de Napierville". 
sur la rive sud du fleuve Saint-Laurent, tandis que le centre de l'armée du nord était a Terrebonne, après quoi on devait s'emparer de Montréal, Trois-Rivières et Québec.

Comme le récìt des événements concurrents du Haut-Canada nous entraînerait trop en dehors de notre sujet, bornons-nous à dire qu'à Beauharnois les Chasseurs se portèrent à l'attaque du Manoir seigneurial de Edward Ellice, sous la conduite de Louis Dumouchelle, du Dr Henri Brien et du notaire Chevalier de Lorimier tandis qu'un autre groupe s'emparait du vapeur Henry Brougham sous les ordres de F.-X. Prieur. A Châteauguay, les Patriotes Cardinal, Duquette, Thibert, Ducharme et Lepailleur constituèrent les bureaucrates de l'endroit prisonniers et se portèrent à Caughnawaga, avec une quarantaine de patriotes armées de fourches et de bâtons, pour s'emparer du dépôt d'armes et de munitions qui s'y trouvait; mais ils furent pris au piège et cernés par les Indiens qui les conduisirent à la prison de Montréal.

Ces attaques eurent une répercussion dans cette dernière ville où l'on arrêta comme suspects Louis-Hippolyte Lafontaine, DenisBenjamin Viger, Jean-Joseph Girouard, Edouard-Raymond Fabre, Pierre Badeaux et autres citoyens marquants, qui furent relâchés quelques jours plus tard sans avoir pu obtenir de mise en accusation ni procès. Viger qui était un avocat bien versé dans les méandres du droit criminel, refusa d'être élargi sans avoir été mis en accusation; mais il ne put réussir dans ses revendications et dut finalement suivre l'exemple de ses compagnons.

Pendant ce temps, on n'était pas inactif au quartier général de Napierville où l'on réunissait tous les Chasseurs de la région; mais il fallait pourvoir au ravitaillement de l'armée improvisée qui s'y trouvait et pour qui le quartier-maître Huot réquisitionnait des vivres soldés de bons du trésor de la République. Cela ne pouvait cependant durer longtemps et, par ailleurs, les Patriotes étaient impatients de se mettre en campagne. Une goélette américaine leur avait apporté 250 fusils, un canon et des munitions à Rouse's Point sur le lac Champlain, mais une compagnie de volontaires était allée les intercepter à Lacolle en s'y retranchant dans un vieux moulin. Côté, Gagnon et les capitaines Grégoire et Morin partirent de Napierville, à la tête d'une centaine d'hommes, pour les déloger et reprendre les armes ainsi perdues; les assiégés, ayant reçu un renfort important de Hemmingford, refoulèrent les Patriotes jusqu'à la frontière américaine avec le double résultat 
que cette défaite faisait perdre au corps d'armée de Napierville des armements précieux et coupait ses communications avec les EtatsUnis.

D'autre part, Sir John Colborne, qui venait d'être remis en charge du gouvernement du pays, par suite du départ de Lord Durham, proclamait la loi martiale, convoquait le Conseil Spécial constitué en remplacement de l'Assemblée Législative et lui faisait adopter une ordonnance à l'effet de suspendre l'acte d'Habeas Corpus. Il constituait en même temps une armée de 7,000 hommes bien équipés et accompagnés d'une batterie de 8 pièces de campagne, en vue d'écraser la petite armée réunie par Nelson à Napierville comme centre du mouvement insurrectionnel.

Outre ce danger qui menaçait terriblement les Patriotes venus de toutes les paroisses où l'évangile de la liberté canadienne avait été prêché; outre la rupture des communications directes avec les partisans des Etats-Unis par suite de la déroute de Lacolle, un incident malencontreux jeta bientôt une nouvelle douche d'eau froide sur l'enthousiasme de la petite troupe. Impatients d'agir et surtout de reprendre contact avec les sympathisants des Etats-Unis, les insurrectionnistes au nombre d'environ 800 , partaient de Napierville le matin du 8 novembre et arrivaient à Lacolle vers les cinq heures du soir, en même temps que Colborne et son armée partaient de Montréal pour le thétre de l'insurrection. Or ils virent arriver, sur les huit heures du soir leur commandant en chef et président Nelson, non pas dans toute sa gloire, mais pieds et poings liés en compagnie de Trépanier et Nicolas, deux de ses principaux lieutenants, sous la garde d'un peloton de ses propres gens qui disaient l'avoir surpris au moment où il se préparait à s'enfuir vers la frontière américaine !

Cette accusation reposait-elle sur un fondement sérieux?

Une déposition faite par Louis Defaillette, un des commandants improvisés d'escouade, lors des enquêtes qui ont été faites par la suite, nous fournit des renseignements assez concluants sur cet incident. Elle a été trouvée dans les papiers recueillis par le notaire Jean-Joseph Girouard et aujourd'hui déposés aux Archives de Québec; on y constate l'anarchie qui régnait dans cette petite armée où un subalterne pouvait, de sa propre autorité, faire arrêter son supérieur sur un simple soupçon personnel et compromettre ainsi tout le succès d'une cause . La scène s'était passée dans la maison de Louis Dupuis, située à faible 
distance de la frontière, où Defaillette avait été mis en charge d'un corps de garde; lisons le récit qu'il nous en fait dans le langage inculte de son témoignage:

\section{DÉPOSITION DE LOUIS DEFAILLETTE AU SUJET DE L'ARRESTATION DE ROBERT NELSON}

Vers quatre heures de l'après-midi, de jeudi avant, ${ }^{5}$ Nelson, François Trépanier père et Nicolas sont venus à notre garde et l'on me dit que Nelson vouloit me parler, je me suis rendu à la maison de Dupuis où il s'étoit retiré pour le voir. Entré dans la maison j'attendois le voir parce qu'il vouloit me voir. $\mathrm{Ne}$ le voyant pas je demandai où est-ce qu'il était. On m'a dit qu'il était au grenier. Je suis monté au grenier et là je l'ai vu avec François Trépanier. Ils étoient assis. Tout de suite Nelson s'est levé et a avancé envers moi pour me donner la main et demander comment ce que je me portois, auquel j'ai dit que je me portais bien. Ensuite je demandai à Nelson pourquoi il était là parce que je soupconnois fortement qu'il était un poltron, car j'ai servi dans la dernière guerre et je trouvais très étrange qu'un Général se mettrait parmi les petites gardes comme il le fesoit. Il me dit qu'il etait là pour nous prendre rejoindre les autres. Se lui ai dit que je ne voulais pas y aller et Là dessus j'ai descendu en bas de la maison laissant Nelson à jaser avec quelques uns qui était monter le voir.

Je suis sorti de la porte et je fis part aux autres d'un dessein que j'avois formé de les faire prisonniers et de les livrer aux troupes et $j$ 'ai réussi à les prendre prisonniers. Nelson et Trépanier avoient entendu à ce qu'il parait qu'on avoit des desseins de la sorte Car Nelson a dardé dans le chassi du sud du greniör mais voyant là un homme avec un fusil qu'il présenta sur lui a reculer et a ensuite dardé dans le chassis de l'autre bord dont il avait cassé la plus grande partie des vitres a ce qu'on m'a dit et j'ai vu les vitres cassés le lendemain. Enfin du monde que j'avois envoyé dans le grenier pour les poigner les ont fait descendre en bas et Là nous les avons lié et attachés les mains de tous les trois derrière le dos. Les trois personnes dont je parle c'est Nelson, Trépanier et Nicholas. Je crois que c'est Joseph Pinsonnault et Fransois Rémillard et d'autres avec, qui les ont attachés. Les prisonniers avaient l'air bien triste. Ils se lamentoient de l'injustice qu'on leur faisoit mais je leur ai dit que cétait par une doutance que j'avais qu'ils étoient là pour nous mettre plus dans la misère.

On faisoit de l'opposition à cette démarche quelques uns des gens n'toient pas en faveur de les faire prisonniers, crainte d'être hachés par les patriotes mais enfin nous sommes partis ayant ces trois prisonniers dans une charette pour aller par le chemin des concessions les livrer aux Troupes à Odell Town. Mais après qu'on avoit fait une petite distance, les gens avoit tant peur de se

5. Soit, le 8 novembre. 
faire hacher par les patriotes qu'ils obtinrent que nous les mènerions au Camp de Lacole et nous sommes rendu là et quelques uns m'ont dit qu'on les avait détacher en route à une petite distance du Camp.

Le lendemain matin, lorsque je me suis parti du Camp pour aller encore à la garde, j'ai vu Nelson. Je ne l'ai point vu depuis. Je me suis rendu à la garde encore par l'ordre de Trudeau. Je dois dire que j'avois envoyé, pendant que nous détenions Nelson et les autres prisonniers, un nommé Lavaller pour avertir les troupes de venir les chercher qu'on les avoit prisonniers, mais je ne l'ai point vu depuis. Mais je crois que les troupes n'ont pas voulu se fier à lui.

Je crois et je croyais dans le temps que le dessein de Nelson était de nous en servir de garde sur sa route dans les Etats pour éclaircir le chemin à lui.

Je n'ai point pris de serment secret, ni n'ai pas été aux assemblées et je ne sais signer.

Dans son numéro du 25 mars 1840 le journal North American auquel collaborait le Dr Côté déclare que cette affaire fut montée par le faux patriote Capitaine Heffernan; mais, d'autre part, Hindenlang a fait grand état de cette supposée trahison du commandant en chef dans sa délation dont nous parlerons plus loin.

Quoi qu'il en soit, Nelson n'eut guère de peine à se disculper;il établit qu'un général dépourvu d'assistants est obligé de voir par luimême à la distribution de ses ordres et des dispositions à prendre pour la bataille; il déclara que, dès le lendemain, il se mettrait à la tête des combattants et son éloquence fut si persuasive que tous jurèrent à nouveau de lui rester fidèles jusqu'à la victoire ou la mort.

Dans le même temps, un autre incident de nature différente, mais tout aussi caractéristique de la confusion qui régnait chez ces braves gens entraînés sans préparation suffisante dans un mouvement irréfléchi, se passait à quelques pas de là. Charles Hindenlang étant allé demander au Dr Côté des instructions au sujet de la bataille qui se préparait, celui-ci lui répondit: "Vous n'avez pas d'ordres à recevoir de moi puisque vous êtes général de brigade". Et le jeune sous-officier, bien qu'étonné d'un si rapide avancement, accepta le commandement dont il était ainsi investi à brûle-pourpoint, déterminé à faire son devoìr dans une cause perdue d'avance.

Enfin, le jour d'action décisive arriva; c'était le 10 novembre 1838 et la petite armée s'avança vers Odelltown en trois colonnes, celle de gauche sous le commandement du général en chef Nelson, celle du centre sous les ordres du major Hébert, jeune notaire de Laprairie, et celle de droite dirigée par le général de brigade Hindenlang. 
Trois cents volontaires bien armés s'étaient retranchés dans l'église, sous les ordres du Colonel Taylor, avec une pièce de canon à leur disposition. L'aile gauche des Patriotes s'embusquait derrière une clôture construite partie en pierre et partie en bois et l'aile droite derrière une grange, alors que la colonne du centre s'avançait à découvert dans le chemin.

Dès la première décharge des volontaires, le cheval du major Hébert, blessé d'un boulet de canon, renversa son cavalier et se sauva a travers les champs. Voyant le danger que couraient ses hommes en rase campagne, Hébert se releva et les divisa en deux groupes qui se joignirent aux ailes latérales et entretinrent un feu nourri sur les loyalistes jusqu'à épuisement de leurs munitions. Au bruit de la fusillade, un renfort de volontaires arriva de Caldwell Manor, mit le feu à la grange et se prépara à cerner les deux groupes de Patriotes avec le concours des soldats sortis de l'église. S'étant consultés hâtivement, les officiers de l'armée d'attaque donnèrent l'ordre de retraiter afin d'éviter une plus grande effusion de sang et les $\mathrm{Pa-}$ triotes se dispersèrent, la plupart d'entre eux cherchant leur salut vers la frontière américaine située à faible distance. Le combat avait duré trois heures.

Hindenlang, qui avait combattu bravement avec sa petite brigade, fut au nombre de ceux qui se rendirent à la merci du vainqueur, tandis que Hébert réussit à atteindre la frontière après avoir erré toute la nuit dans les bois et s'être gelé les pieds. Quant au Dr Nelson, président de la République Canadienne et commandant improvisé des troupes, une déposition de Louis L'Huissier, trouvée dans les papiers Girouard, nous dit qu'il s'est enfui a bride abattue vers les Etats-Unis dès la première décharge d'artillerie, tandis que ses panégyristes prétendent qu'il "courut chercher des médicaments pour soigner ses blessés". Un fait certain, c'est qu'il put atteindre la frontière et se mettre à couvert au lieu de partager le sort des prisonniers.

On ne connaîtra probablement jamais l'exacte vérité à ce sujet. A-t-il cédé à la terreur irraisonnée des conscrits qui subissent le baptême du feu, et lâchement abandonné ses soldats, ainsi que fit le "général" Amury Girod à la bataille de Saint Eustache? A-t-il tout simplement voulu se protéger contre l'emportement de ses propres gens qui voulaient lui faire un mauvais parti lorsqu'ils constatèrent que l'aventure dans laquelle il les avait entraînés tournait au tra- 
gique? A-t-il plutôt résisté jusqu'au bout avec une poignée de braves et fui vers la protection du drapeau étoilé seulement après avoir constaté que tout était perdu? Cherchons la réponse à ces questions dans les documents que nous avons consultés.

La confession d'Hindenlang, que nous rapportons ci-après, ferait admettre la première hypothèse si elle ne portait pas l'empreinte d'un accusé qui cherche à obtenir sa grâce en dénonçant ses complices et qui exhale son amertume à l'adresse de ceux qui l'ont conduit sur le chemin de l'échafaud. Cette "déclaration volontaire" faite à la prison de Montréal dans le gilet d'un geôlier quatre jours après la déroute d'Odelltown, est celle d'un participant qui se voyait dans une terrible impasse et qui cherche évidemment un bouc émissaire de tous les péchés d'Israël; elle a plutôt le caractère d'une diatribe que d'un acte de contrition. Lisons-la pour nous en convaincre; elle fut publiée dans l'Ami du Peuple, journal bureaucratique de Montréal et reproduite en placard pour plus grande diffusion avec le commentaire suivant: "Nous publions ici la relation donnée par $M$. Chs. Hindenlang, l'officier français que les rebelles avaient décoré du nom de général. Cette courte et simple narration d'un homme aussi horriblement trompé par les rebelles doit ouvrir les yeux à tous les Canadiens qui voudront la lire sans préjugés."

\section{DECLARATION VOLONTAIRE DE M. CHARLES HINDENLANG Général de Brigade dans l'armée des rebelles}

“Je suis né à Paris le 29 mars 1810... Lors de notre glorieuse révolution de 1830, malgré ma mère, je pris du service comme simple soldat au 5è régimeñt d'infanterie légère. Je passai successivement par tous les grades jusqu'à celui d'officier. Mais voyant peu d'avancement possible dans l'armée, mes parents m'engagèrent à quitter entièrement le parti des armes et à venir à New York où leur intention était de m'envoyer des marchandises au printemps prochain. J'étais fort tranquille, ne songeant qu'à me mettre au courant des affaires de cette ville lorsque $M$. Duvernay me fit successivement demander par plusieurs personnes entre autres par un Italien réfugié nommé Fälière qu'il voulait avoir aussi pour officier. J'ai été également demandé, et j'ai même connu, pendant quelques jours, un M. Von Schoultze, se disant colonel et cherchant des officiers et soldats pour le Haut Canada.

...M. Duvernay agissant au nom et pour le Docteur Nelson (Robert) me fit à moi et à un autre officier français qui a servi dans le 1er lanciers anglais en Espagne en qualité de lieutenant ou capitaine des propositions et de belles promesses en présence d'un négociant nommé $M$. Bonnafous... Après nous être 
consultés nous refusâmes de signer avant d'être mieux informés de la situation du Canada.

...Alors pour mieux nous tromper l'on nous assura qu'il ne s'agissait que d'aller à St. Albans former des cadres d'organisation pour l'armée....Non seulement le Peuple Canadien se, souleverait en masse, mais la troupe et surtout messieurs les officiers anglais étaient las d'un service si éloigné de leur patrie et n'attendaient que cette occasion d'en sortir.

...Nous décidâmes à aller nous assurer par nous mêmes de la vérité de tout cela. Nous fûmes donc dirigés sur Burlington et reçus par M. Dufort. Toujours même langage et mêmes promesses... M. Dufort nous expédia à Plattsburg où nous trouvâmes des chevaux et fûmes conduits par ce qu'ils appellent un chasseur jusqu'à Champlain, chez M. Desmarais qui nous tenait cachés comme si nous étions de grands coupables. En général j'ai remarqué chez tous ces agents une pusillanimité qui allait jusqu'à la faiblesse, pour ne pas dire davantage.

Nous demandâmes à être conduits, suivant la promesse, à St. Albans; l'on nous fit passer la rivière en chaloupe et de l'autre côté, nous trouvâmes le docteur Nelson et un Canadien que je suppose être un guide. Cette nuit même, le $^{2}$ peuple canadien soulevé devait, suivant l'infâme et misérable Nelson, se trouver sur les côtes pour le recevoir et le féliciter de son débarquement. 250 fusils avaient été amenés et déposés dans ma chaloupe par le colonel américain Burton! Nous partîmes et nous descendímes la rivière jusqu'au quai Vitman, le docteur, son guide, un pilote et nous deux. Nous arrivons par un temps affreux; pas un seul homme pour recevoir le fameux président du gouvernement provisoire et ce n'est qu'après une grande heure d'attente et de misère que le guide revint avec quelques hommes (5 ou 6 au plus) pour débarquer les fusils. A l'instant on nous donna 3 chevaux et nous fîmes route pour Napierville, c'est là que le docteur Côté, à la tête de $\mathcal{Q}$ ou 300 hommes reşut le Dr. Nelson et le proclama président de la république du Bas Canada... c'était le dimanche matin (4 novembre 1838).

Pendant trois jours, le Dr. Nelson et son collègue ne s'occupèrent guère que de messages pour hâter l'arrivée des hommes. Le lundi, l'officier français Touvrey partit avec 50 hommes pour sonder et éclairer les environs; depuis, je ne l'ai pas revu.

...Le même soir, une dame me fit demander si je voulais faire remettre un lit et un oreiller à un prisonnier âgé et malade; je le fis à l'instant et, à mon retour, je crus devoir le communiquer au Dr. Côté. C'est alors qu'il me prit en particulier et qu'il me dit que je n'avais pas d'ordres à recevoir de lui etque j'étais brigadier... Faire un général comme on fait un soldat! je n'ai pu m'empêcher d'en rire avec plusieurs personnes...

Le lendemain, le Dr. Côté partit avec 50 ou 60 hommes armés, plus un autre détachement envoyé pour renforcer celui commandé par l'officier Touvrey; j'ai su depuis que less armes que le Dr. Côté voulait faire entrer avaient été saisies, qu'il avait été repoussé par les troupes de la reine en laissant un canon entre leurs mains. 
Pendant ces trois jours, des Canadiens armés et non armés, pressés par les messagers du Dr. Nelson se rendaient à Napierville et j'estime à Q,500 hommes la force générale qui a pu se réunir à cet endroit... Le jeudi le Dr Nelson commanda la marche sur Odelltown, 600 hommes armés et tous les officiers l'accomcompagnaient... La pluie nous arrêta à Lacolle où nous passâmes la nuit; c'est là que, sous le prétexte d'aller rejoindre, pour l'amener, un poste éloigné de 150 hommes commandé par un nommé Dupuis et un autre capitaine, le lâche et misérable Nelson, muni de tout l'argent qu'il put rassembler, chercha à se sauver; il fut lié, garotté et sur le point d'être livré par les Canadiens; il ne dut son salut qu'aux prières et à l'intercession des capitaines Nicolas et Trudeau.

Il revint à Lacolle où par les plus grands serments, il parvint encore à faire croire à sa franchise, il décida que le lendemain une attaque conduite par lui-même en " personne aurait lieu sur Odelltown. Je jure sur l'honneur qu'un grand nombre d'hommes ont èté forcés par les menaces et les précautions prises par le lâche Nelson, de suivre le mouvement; il fallait se déclarer patriote ou bureaucrate. La peur a fait bien des patriotes malgré eux.

...En arrivant sur le plateau faisant face à la maison fortifiée, la plus grande partie des hommes se répandit dans la plaine à droite; sur la gauche, il n'y eut qu'une poignée d'hommes courageux qui furent s'embusquer dernière une grange et commencèrent le feu. La plus grande partie des Canadiens était hors de la portée du fusil, à genoux, le visage dans la neige, priant Dieu et ne remuant pas plus que des saints de pierre; plusieurs restèrent dans cette position tout le temps que dura le feu. Oh ! pitié pour de tels hommes; il a fallu être bien fourbe et bien adroit pour amener à la révolte de semblables gens!

...Alors je fus enfin convaincu que le Dr. Nelson n'était qu'un lâche et vil moteur de troubles, qu'un voleur éhonté qui fuyait avec l'argent, laissant massacrer des gens qui, sans ses perfides insinuations, seraient tranquilles dans leurs chaumières dont il ne reste plus que la place...

...Mon parti était pris: sauver des malheureux s'il était possible; je courus la plaine demandant au milieu des balles ce qu'était devenu le Dr. Nelson;... le misérable avait profité du moment où les troupes étaient occupées pour gagner la frontière par un détour. Je restai paisible spectateur de l'action, n'attendant que le moment de pouvoir me livrer et traiter du salut de tous ces malheureux... J'arrivai des derniers à Napierville; les officiers étaient assemblés et s'occupaient de placer des gardes. C'est alors que je signifiai hautement que, ayant été trompé et pouvant juger par moi-même de toute la stupidité d'une pareille entreprise... Je me regardais comme entièrement libre de mes actions et nullement lié à la cause des malheureux Canadiens.

...Nous partîmes une douzaine ensemble. Nous prîmes les bois pour éviter les postes de nos gens qui nous eussent arrêtés et, au point du jour... je sortis $d u$ bois et fus me rendre à un jeune homme que je vis au loin, armé d'un fusil... Enfin, à Lacolle, je demandai à des officiers la permission d'écrire une lettre $\grave{a}$ Son Excellence... mais ce n'est qu'aujourd'hui que je puis exposer tout ce que je puis savoir. C'est une haine et un mépris profond qui m'animent contre le Doct. Nelson et ses complices. 
Je n'ai pu voir, sans frémir de colère, un seul homme méditer de sang-froid et avec connaissance de cause, la perte de tout un peuple et cela par un vil intérêt. Je tiens de lui-même que la seule ville de Montréal lui a envoyé plus de \$0,000 piastres. Qu'a-t-il pu faire de cet argent?

...Pendant 5 jours, je me suis vu dans les rangs des ennemis d'un gouvernement ami et allié de ma patrie. Voilà ma faute, rien ne me cô̂tera pour la réparer;... que l'on m'emploie au service de la bonne cause et j'engage ma parole d'honneur de réparer, par tous les services possibles et exigibles, quelques jours d'égarement.

Je jure sur l'honneur et devant Dieu que tout ce que j'ai écrit est vrai... Je jure aussi d'être toujours l'ennemi acharné du Dr. Nelson, de le poursuivre jusqu'à ce que je puisse lui reprocher son infamie devant le monde entier, si c'est possible.

Fait à la prison de Montréal, ce 14 novembre 1838

Chs. Hindenlang

Reconnu devant moi, en la prison de Montréal, ce 14 novembre 1838

P.E. Lechèrc, J.P.

Cette longue confession pro domo est appréciée comme suit par l'Estafette, journal français publié a New York: " $M$. Hindenlang, enrôlé a New York, dans la cause canadienne, a été une des premières victimes de la nouvelle insurrection. Il s'est trouvé l'un des premiers face à face avec la potence - et il a eu peur! Les promesses de ses géoliers, ou seulement peut-être l'espoir d'obtenir grâce, lui ont fait faire une longue et lamentable confession." De son côté le Canadien, journal publié à Québec par Etienne Parent, disait en rapportant la déclaration d'Hindenlang à la date du 23 novembre 1838: "Cette lettre d'Hindenlang fut amplifiée et corrigée par P.-E. Leclerc."

Mais ce n'est pas tout. Dans le but de donner plus de force à sa dénonciation du Dr Nelson, et sans doute aussi pour confirmer l'accusation de tromperie dont il prétendait avoir été victime, avec l'officier Touvrey, de la part des insurgés canadiens, Hindenlang écrivait à l'adresse de celui-ci ainsi qu'à un ami désigné sous le nom d'Henri, deux lettres non datées (portant simplement l'indication - "Nov. 1838") contenant les mêmes diatribes sur le compte de Nelson et ces lettres furent apparemment remises au geôlier Leclèrc pour appuyer sa confession. Nous ne savons qui était cet "Henri" ni si sa lettre lui est parvenue, mais elle fut publiée dans les journaux, en même temps que celle adressée à Touvrey qui y répondit vertement comme on 
pourra le constater ci-après. Voici les extraits essentiels de ces documents.

\section{LETTRE DE HINDENLANG A TOUVREY}

(datée de la prison de Montréal le - Nov. 1838)

Mon cher ami,

"Nos tristes pressentiments sont accomplis. Nous avons été lâchement trompés par le docteur Nelson, que n'avons-nous écouté nos premières pensées de méfiance; vous le savez après avoir essayé déjà une fois de se sauver avec tout l'argent qu'il a pu se procurer, le lâche déloyal docteur a abandonné honteusement tous les gens qu'il conduisait au feu... nos deux braves polonais sont avec vous... Retournez à New-York... poursuivez l'homme déloyal qui m'a perdu, demandez lui compte de ses mensonges, de sa déloyauté et de son abandon"etc.

Chs. Hindenlang

\section{LETTRE DE HINDENLANG A HENRI (?) le-Nov. 1838}

..."La Lâcheté du Dr. Nelson qui a pris la fuite en abandonnant tous ses gens et emportant tout l'argent t'est sans doute déjà connue. Garde-toi bien, mon ami, de prendre part à tous les mouvements qui pourront avoir lieu; que l'exemple de ton ami te serve de leşon" etc...

P.S. "Mes amitiés à Vignos, M. Goll et autres. Si tu apprenais quelques malheurs, sois l'interprète de mes derniers adieux à Anna, rue de Cléry, 47 ou 41, Ecris de suite à mon frère par le prochain courrier, 19 rue des Vinaigriers".

Chs. Hindenlang;

Passons maintenant à la deuxième conjecture de la supposée fuite du Dr Nelson à Odelltown parce qu'il redoutait l'emportement de ses propres gens. Elle s'appuierait sur une lettre du Dr Davignon à Sydney Cowen, écrite à Rouse's-Point en date du 12 novembre 1838 (deux jours après cet événement), qui nous est rapportée comme suit dans le journal d'Amédée Papineau. ${ }^{6}$

\section{RÉCIT DU DR. FRANÇOIS-JOSEPH DAVIGNON.}

"Le soir après la bataille, Nelson adressa une lettre à l'armée dans laquelle il déclare qu'il craint d'être assassiné par ses propres soldats et qu'il résigne la présidence, mais sans abandonner la cause qu'il espère encore servir aux

6. Journal d'un Fils de la Liberté, etc. à la date du 17 novembre 1838. 
États-Unis ou dans le Haut Canada. La-dessus, les Patriotes se sont débandés et sont très mécontents contre Nelson et Coté...

On a fait croire aux Patriotes en Canada, que M. Papineau était à la tête de ce mouvement. Sans cela, ils ne se seraient pas soulevés. Cette fourberie les a découragés et fait disperser. Cest pourquoi aussi plusieurs canadiens, voulant connaître l'opinion de M. Papineau, se sont assemblés ici et lui ont écrit, lui demandant son avis et le priant de faire son possible pour les retirer du mauvais pas où on les a jetés."

Cette crainte d'un mauvais parti aux mains de ceux qu'il avait entraînés (avec le Dr Côté du reste) dans cette échauffourée expliquerait pourquoi le Dr Nelson n'est pas revenu s'établir au Canada après l'amnistie générale des révoltés, à l'exemple de son frère Wolfred qui a joué par la suite, un rôle important dans les affaires de son pays, ayant même été élu député du comté de Richelieu contre l'honorable D.-B. Viger, et maire de Montréal à deux reprises. Au lieu de revenir alors parmi ses compatriotes qui l'avaient autrefois élu deux fois député de Montréal-Ouest avant l'insurrection, Robert Nelson est allé planter sa tente à Los Angeles en Californie, loin de ses anciens compagnons d'armes, où son habileté professionnelle lui a permis d'acquérir une belle aisance, et il est revenu à New-York où il est décédé (à Staten Island) en 1873, à l'âge de 80 ans.

La troisième supposition de l'attitude exacte du commandant Nelson à la bataille d'Odelltown s'appuie sur une lettre de l'officier Touvrey, embauché dans l'invasion canadienne en même temps que Hindenlang et que celui-ci appelle en témoignage dans sa confession. Cette lettre est adressée au journal l'Estafette en date du 29 novembre 1838, corroborée par l'officier polonais Oklowski, que nous reproduisons également ci-après, et elle fait entendre une toute autre note. Loin de faire écho aux accusations de Hindenlang, Touvrey le rabroue d'importance et venge le Dr Nelson des injures de "voleur" et de "lâche" que celui-ci lui verse à pleines pages.

Voici les principaux passages de cette lettre historique dont l'existence a paru échapper à la plupart de nos écrivains, de même que la personnalité de son auteur. Publié par un journal de New York en réponse à celle que Hindenlang lui avait adressée par la voie de certains journaux du Canada, elle fut reproduite par le Canadien, journal d'Etienne Parent à Québec, en date du 10 décembre 1838, avec le commentaire suivant au sujet de Hindenlang: "Il a été repoussé par ceux dont 
il demandait grâce; il a excité le dégoût de tout le restant de la population et voild qu'il est honni et démenti par ses propres amis et compatriotes. Avis aux délateurs".

\section{LETTRE DE PH. TOUVREY EN REPPONSE A L'APPEL DE CHS. HINDENLANG}

\section{A l'Éditeur de l'Estafette de New-York.}

" $J$ 'ai lu avec le plus grand étonnement, sur votre feuille de mardi dernier, une lettre entre plusieurs autres qui m'est personnellement adressée et qui ne m'est parvenue que par la voie des journaux.

...On sait jusqu'où peut se porter la lâcheté de certains gens qui ont à choisir entre la délation ou l'échafaud.

...J e crois donc de mon devoir de contredire volontairement les faux avancés, les calomnies insignes rédigées et publiées par les TOREES de Montréal sous la signature de Charles Hindenlang... cette déclaration fut faite devant le chef de police P.E. Leclère (un diminutif de Vidocq) et contresignée par cet individu. Si on en croit la renommée, ce contreseing est plus que suffisant pour jeter du louche sur cette déclaration et les différentes lettres qui ont été publiées.

...Il (Hindenlang) ne vint pas en Amérique pour des affaires de commerce, comme il l'a déclaré sous serment mais bien pour joindre las patriotes du Canada. A la première nouvelle qui arriva à Paris l'hiver dernier, de l'insurrection canadienne, il s'embarqua avec le nommé Henri, le même à qui il a adressé aussi une lettre dernièrement.

...La déposition et les lettres de Chs. Hindenlang sont remplies d'injures, d'autant plus atroces qu'elles ne sont pas méritées, contre les patriotes et leurs chefs, et particulièrement contre le Dr. Robert Nelson. Je puis dire que j'ai été plus à portée de connấtre ce qui s'est passé à Napierville et aux deux combats de Lacolle et de ODLETOWN que M. Chs. Hindenlang, et pour cause! Ainsi mon témoignage, appuyé de l'officier polonais qui a aussi pris part dans ces affaires, devra avoir quelqu'influence sur le public... Tous les fonds collectés d'avance avaient été déposés entre les mains d'un trésorier qui les avait employés à l'achat et au transport des armes et des munitions... Une autre preuve que le Dr. était sans argent est qu'il fut arrêté par les chefs d'une conspiration formée contre lui et que le Dr. fut saisi, fouillé et désarmé. Ainsi, s'il eût eu sur lui $\$ 1,500.00$ en espèces, ceux qui l'arrêtèrent n'eurent pas manqué de s'en saisir et de se les approprier.

Le titre de LACHE que le délateur Hindenlang prodigue au Dr. Nelson n'est pas plus mérité que celui de VOLEUR... Je puis prouver que le Dr. N. ne laissa ODLETOWN que quelques minutes avant la fin du combat, et cela pour tâcher de se procurer ce qui était nécessaire aux blessés qui réclamaient ses soins. Il ne se trouvait pas un autre chirurgien sur les lieux. Un détachement de $\mathbf{3 0 0}$ hommes de troupes frâ̂ches qui arriva, empêcha le Dr. de rejoindre les siens. D'ailleurs le combat avait cessé. 
La même accusation lancée contre les Canadiens en général est aussi dépourvue de fondement. J'ai pris part à quantité de combats, en France, en Espagne, et en Portugal et je puis dire avec vérité que les Canadiens n'en cèdent pas aux autres peuples quant à la bravoure et à l'activité. On sait que les Canadiens sont sans expérience et sans tactique dans l'art de la guerre; ainsi il était impossible de s'attendre qu'ils dussent combattre comme d'anciens militaires... Que l'on fournisse aux Canadiens des armes des munitions et des chefs versés dans l'art de la guerre et en bien peu de temps, on peut en faire d'excellents soldats... M. Hindenlang aurait dû s'épargner la peine de parler de ce qui a pu se passer durant le combat puisqu'il ne l'a vu qu'à distance !

...Il y a contradiction palpable dans toutes les productions de M. Hindenlang, ce qui prouve qu'il n'en est pas lui-même l'auteur.

...En dépit des conseils de mon bon ami Mr. Hindenlang, qui me prie d'abandonner une cause perdue et de me tenir éloigné des voleurs, des lâches et des brigands, je suis prêt à m'enrôler de nouveau sous la bannière de la bonne cause, celle de la liberté.

...En résumé, je déclare que d'après la connaissance personnelle que j'ai de tous les faits, la déclaration et les lettres de Chs. Hindenlang sont des tissus de mensonges et d'atrocités dignes d'un trâ̂tre et d'un délateur.

Croyez-moi Mr. L'Editeur

$$
\text { Votre dévoué serviteur }
$$

Ph. Touvrey

New York, 29 novembre 1898.

Après avoir pris connaissance de la lettre précédente, je déclare qu'elle contient la vérité.

\section{Ferdinand Alfhonse Oklowski Officier polonais}

Ayant lu ces divers documents, sommes-nous définitivement fixés sur l'accusation de lâcheté lancée contre le président de la République du Bas-Canada et commandant en chef de l'armée des Patriotes a la bataille d'Odelltown? Nous ne le croyons pas, - pas plus que nous ne sommes fixés sur la controverse qui s'est élevée entre Wolfred Nelson et Louis-Joseph Papineau, par suite du fait que celui-ci s'est tenu à l'écart de la bataille de Saint-Denis en 1837. Dans le doute, il vaut mieux donner acte à l'un et à l'autre de la sincérité de leurs revendications patriotiques et louer le Dr Robert Nelson de sa persistance à en poursuivre la réalisation, de même que de son audace à tenter avec une poignée de partisans la libération de sa patrie. S'il eât réussi, son nom serait inscrit au pinacle de l'histoire canadienne à côté de celui de Papineau qui a conduit la lutte constitutionnelle au Parlement pendant un si grand nombre d'années. 
Revenons maintenant aux commandants des deux autres ailes de l'armée républicaine du Bas-Canada, le major Hébert et le général de brigade Hindenlang. Le premier, qui, avant cette investiture, était un tout pacifique tabellion exerçant à Laprairie, dut se trouver fort désemparé, s'il est vrai que ses soldats, au témoignage de Hindenlang, "se jetaient à genoux dans la neige pour implorer le Ciel" au lieu de combattre. Il faut bien se rappeler que la moitié de ces soldats improvisés n'étaient armés que de fourches et de bâtons; dans un combat d'armes à feu, ils n'avaient d'autre ressource que de se coucher à plat ventre pour essuyer les décharges de mousqueterie en attendant le moment de monter à l'assaut ou de ramasser les fusils des morts. "Pitié pour de tels hommes" écrivait Hindenlang; admirons plutôt la sublimité de leur courage dans une situation désespérée et rappelonsnous que Touvrey leur a rendu hommage dans la lettre que nous avons reproduite ci-dessus.

Médard Hébert, né en 1810, avait fait ses études légales chez Louis Barbeau, notaire à Laprairie, dont il épousa la fille et s'établit au même endroit. Réfugié aux Etats-Unis à la suite de la bataille d'Odelltown, il ouvrit une épicerie à Plattsburg, en société avec son confrère, le patriote Demaray, ${ }^{7}$ afin de se procurer des moyens de subsistance pendant leur séjour en terre étrangère. Revenu au Canada en 1841, à la faveur d'une amnistie, il reprit l'exercice de sa profession à Laprairie où il mourut en 1849.

Quant à Hindenlang (dont les manuels d'histoire s'obstinent à orthographier le nom "Hindelang"), nous connaissons le pénible sort de ce jeune enthousiaste de 28 ans, épris des principes libertaires de la Révolution française, mais fourvoyé en pays étranger dans une aventure vouée à l'insuccès. On n'a pas lu sans émotion le discours empoignant qu'il a prononcé au "souper des Girondins" canadiens ${ }^{8}$ lorsque les condamnés à la potence dirent adieu à leurs compagnons de captivité le 14 février 1839, veille de leur exécution; aussi ne peuton s'expliquer la pusillanimité de l'offre, contenue dans sa confession, de réparer, si on lui faisait grâce, "par tous les services qu'on exigerait de lui, ou qui seraient en son pouvoir, ses quelques jours d'erreur ou d'égarements."

7. Celui qui avait été libéré par Bonaventure Viger sur le chemin de Chambly, en compagnie du Dr Davignon, au commencement des troubles de 1837. 1913).

8. Cf. L.-O. David, Les Patriotes de 1887-1838. (Montréal, Librairie Beauchemin 
A-t-il vraiment écrit ces paroles? Il est permis d'en douter, car nous avons le témoignage du journal d'Amédée Papineau déclarant, à la date du 25 novembre 1838, que "cette lettre fut amplifiée et corrigée par P.E. Leclerc". Cette amplification avait assurément pour but de chercher à sauver celui dont elle portait la signature, mais elle ne pouvait manquer de porter atteinte à la réputation de son auteur putatif dans la critique impartiale de l'histoire.

Hindenlang a payé de sa vie la participation qu'il a prise aux revendications libertaires du peuple canadien; son sort lui a mérité le respect qu'on doit à ceux qui se sacrifient pour une grande cause. Il avait donné son corps au Dr Vallée en demandant de lui donner la sépulture et d'envoyer son cœur à sa mère, à Paris, dans un bocal d'essence de vin, mais ce désir n'a pu être exaucé. Il a été inhumé dans le cimetière militaire de la rue Papineau.

Il nous reste à dire un mot de quelques autres chefs dont les noms figurent au nombre des principaux acteurs de ce drame.

Né à Québec en 1809, le Dr Cyrille-Hector-Octave Côté s'était tout d'abord établi comme médecin à l'Acadie en 1831, puis s'était fixé à Napierville en 1833. Il fut élu député du comté de l'Acadie l'année suivante et prit une part importante au soulèvement de 1837. Réfugié aux Etats-Unis après la bataille de Saint-Charles, il s'occupait activement à préparer la revanche; c'est pourquoi on le retrouve à tous moments dans les péripéties de l'invasion de 1838.

De retour aux Etats-Unis après l'échec de son coup de main à Lacolle, il s'établit à Swanton où il séjourna jusqu'à sa rentrée au Canada comme bénéficiaire de l'amnistie générale de 1844. Il avait apostasié en 1841 et s'était même fait admettre ministre baptiste; c'est en cette qualité qu'il chercha à faire du prosélytisme à SaintPie-de-Bagot et qu'il se fixa définitivement à la mission de la Grande Ligne, près de la frontière américaine, où il fut inhumé en 1850.

Le Dr J.-B.-Henri Brien, établi à Sainte-Martine, s'était réfugié aux Etats-Unis après l'échec du soulèvement de 1837. Il prit part à la préparation de la revanche de février 1838 en dirigeant la fabrication de 80,000 cartouches à Plattsburg; mais, vu l'avortement de la première tentative d'invasion de février 1838 , il revint pacifiquement au Canada le 5 juillet suivant.

Appelé par les Patriotes à seconder Dumouchelle dans l'attaque du 3 novembre à Beauharnois, il cessait toute participation aux 
opérations dès le lendemain et se dirigeait de nouveau vers les EtatsUnis, lorsqu'il fut arrêté et conduit à la prison de Montréal où il commençait, le 18 novembre 1838, la rédaction d'un mémoire historique des événements auxquels il avait pris part ${ }^{9}$. Traduit en cour martiale pour haute trahison devant le tribunal présidé par le major-général Clitherow, il fut condamné à la potence, en même temps que Chevalier de Lorimier, Dumouchelle, Prieur et autres accusés, après un procès qui dura du 11 au 21 janvier 1839; mais sa sentence fut subséquemment commuée en un bannissement à 600 milles du Bas-Canada. ${ }^{10}$

De Lorimier, Cardinal, Duquette et leurs malheureux compagnons ont expié sur l'échafaud l'erreur de s'être crus en mesure de libérer leur pays de l'oppression administrative par la force armée. D'autres ont répandu leur sang sur les champs de bataille pendant que les incendies allumés par la soldatesque irritée détruisaient les foyers de leurs familles. ${ }^{11}$ Rendons hommage à leur mémoire.

Mais peut-on dire que toutes ces calamités ont été subies en pure perte?

Tout au contraire, le sang des martyrs n'est jamais stérile. Si les soulèvements de 1837 et 1838 n'ont pas immédiatement produit les résultats que leurs meneurs en attendaient, ils ont fait comprendre en haut lieu qu'il y avait des abus à corriger. Un envoyé extraordinaire, Lord Durham, fut délégué parmi nous afin de se renseigner sur place et son rapport, bien qu'entaché de quelques erreurs d'appréciation mais surtout de tentatives d'écrasement de notre race, a déterminé des réformes qui ont conduit à la conquête de nos libertés politiques.

Depuis 1791, nous jouissions théoriquement d'un régime parlementaire calqué sur celui du gouvernement britannique; mais une

9. Ce mémoire, rempli de détails intéressants, a été publié par la Société d'Archéologie de Montréal au Vol. 5 de la troisième série du Canadian Antiquarian.

10. Cf. State Trials, Vol.2, Index.

11. Les sinistrés se sont vengés de ces représailles en attribuant à Sir John Colborne le qualificatif de "Vieux Brûlot". 
oligarchie puissante infirmait en pratique les avantages que le peuple en avait escomptés dans l'administration de la chose publique. Continuellement rebutés dans leurs justes revendications, nombre de chefs crurent à un appel aux armes comme à un dernier recours; ils affirmeraient ainsi, pensaient-ils, leur détermination de poursuivre jusqu'au désespoir une lutte engagée dans l'enceinte des parlements et dans les assemblées populaires, mais qui leur semblait vouée à l'insuccès. Pendant ce même temps, les têtes pondérées avaient plutôt confiance de conquérir la reconnaissance de nos droits par la logique et la stratégie parlementaires.

Dès l'origine du mouvement insurrectionnel, à l'assemblée de Saint-Charles, Papineau dont l'éloquence entraînante avait électrisé les foules, vit avec effroi les conséquences de l'incendie qu'il avait allumé; il conseilla de s'en tenir aux bornes de l'agitation constitutionnelle, tandis que Wolfred Nelson, Rodier, Côté et leurs fougueux partisans sonnaient l'appel aux armes. William-Lyon Mackenzie et Joseph Howe organisaient de semblables levées de boucliers dans le Haut-Canada et la Nouvelle-Ecosse, avec les mêmes résultats douloureux que nous avons indiqués: leurs têtes et celles de nombre de leurs partisans furent mises à prix. Mais, lorsque la bourrasque fut calmée, la plupart d'entre eux sont revenus au timon des affaires du pays et ils ont eu la satisfaction de voir se réaliser le plus grand nombre de leurs revendications, en particulier celle de l'octroi du gouvernement responsable.

Quatre ans après le soulèvement de 1837, Louis-Hippolyte Lafontaine reprenait la thèse de Papineau dans l'enceinte du Parlement d'Union des Canadas avec le concours de Robert Baldwin, ancien membre du cabinet du Haut-Canada. Tous deux étaient restés à l'écart de l'insurrection armée, mais ils réclamaient, par voie constitutionnelle, les mêmes réformes que leurs bouillants amis. Ils n'y réussirent pas encore cette fois; pourtant l'heure du triomphe arrivait enfin en 1848, alors que la ténacité, l'éloquence, l'habileté et la stratégie parlementaire de Lafontaine, de Baldwin et de leurs compagnons, appuyées de la largeur d'esprit et de la clairvoyance du gouverneur général, Lord Elgin, finissaient par conquérir à notre profit cette réforme d'un gouvernement du peuple, par les représentants du peuple et pour la sauvegarde des droits du peuple. 
On a diversement apprécié, sous l'empire des passions déchaînées aux heures de la tourmente, la conduite des principaux acteurs du drame que nous venons d'évoquer rapidement. Le recul des événements nous permet évidemment de les juger avec plus d'impartialité, mais un fait brutal ne peut manquer de nous frapper: c'est que les impulsifs, les exaltés, ceux qui activent le feu de la révolte produisent rarement des œuvres constructives, tandis que les sages qui calculent froidement les risques et les chances de succès d'une entreprise importante et qui, tout en en préparant les voies, savent choisir le moment propice d'agir avec discernement, sont plus en mesure d'assurer la pérennité du résultat de leurs travaux. Ainsi, les deux Nelson, dont le patriotisme était assurément sincère mais le zèle irréfléchi, en déclenchant sans préparation suffisante les deux insurrections de 1837 et 1838, n'ont abouti qu'à de lamentables échecs. Papineau, élevé sur le pavois par une foule en délire, n'a pu continuer sur le terrain constitutionnel une lutte qu'il y avait amorcée mais qu'il s'est vu forcé d'abandonner lorsqu'il fut entraîné par ses amis dans la tourmente. LaFontaine, au contraire, a attendu son heure; impassible en face des vociférations de ses ennemis, pondéré, stratège et tenace dans ses revendications, il a vraiment accompli pour son peuple la conquête de sa liberté.

Relisons à ce sujet deux appréciations du rôle et de l'œuvre de ces hommes; l'une exprimée par M. de Pontois, ambassadeur de France aux Etats-Unis, dans une lettre adressée au comte Molé, président du Conseil et ministre des Affaires Etrangères de France, en date du 30 novembre 1838, ${ }^{12}$ et l'autre de notre ami regretté Aegidius Fauteux dans sa préface au volume publié à l'occasion du dévoilement du monument érigé en l'honneur de LaFontaine à Montréal. ${ }^{13}$

12. Cette lettre est reproduite au fascicule No 2 du Volume IV de Nova-Francia avec une introduction de La Rocque de Roquebrune.

13. Cf. Hommages d LaFontaine, publié par le Comité du monument LaFontaine, Montréal, 1931. 


\section{EXTRAIT DE LA CORRESPONDANCE DE M. L'AMBASSADEUR E. de PONTOIS.}

...M. Papineau est venu me voir il y a quelques jours. J'ai acquis par sa conversation de nouvelles preuves de l'impossibilité du succès de l' Insurrection. "C'est, a-t-il fini par m'avouer lui-même, une population réduite au désespoir, qui se précipite aveuglément au devant du danger, sans concert, sans organisation, sans secours étranger et qui se dévoue à la mort."

J'ai cru devoir lui représenter alors...que si un pareil sentiment pouvait être excusable dans les Masses il ne l'était pas dans leurs Chefs dont le devoir était, au contraire, d'user de tous les moyens en leur pouvoir pour arrêter une lutte trop inégale... C'était une prompte soumission, ai-je ajouté, qui pouvait seule donner au Gouvernement de la Reine la possibilité d'écouter les plaintes des Canadiens et de protéger leurs droits et leur Nationalité contre le Parti qui en demandaithautement l'annéantissement ${ }^{14}$ et dont les circonstances actuelles ne favorisaient que trop l'animosité et les exigences.

...J'ai dit enfin à M. Papineau que ce beau et noble rôle de sauveur de ses Compatriotes pouvait, s'il le voulait, lui appartenir à lui qui, je le savais, avait déconseillé l'Insurrection, qui était dénoncé par les Mẹnours actuels, et qui représentait seul, aux yeux de tous les gens éclairés de ce pays, comme à ceux des autorités Anglaisas elles-mêmes, le côté honorable et vraiment patriotique de la cause Canadienne; qu'il ne lui fallait, pour cela, que se séparer, dès à présent et avec éclat, des Intrigants qui s'étaient mis à la tête des derniers mouvements et avaient exploité la crédulité des Canadiens, recommander à ses Partisans la soumission et la patience et, en même temps, réclamer avec énergie et persévérance auprès du Gouvt. Britannique contre le Régime illégal, arbitraire et violent qui opprimait le pays et lui préparait de nouveaux troubles. ${ }^{15}$ ...Malheureusement, Monsieur le Comte, un pareil rôle est au-dessus de la portée de M. Papineau, homme honnête et consciencieux, mais d'un esprit médiocre et étroit, rempli de lieux communs à la place d'idées et opposant à la logique des faits et des réalités de vaines utopies et de puériles illusions, en un mot, précisément le contraire de ce que doit être un Chef de Parti. Au reste il est juste de dire que, voulut-il adopter le Plan que je lui indiquais, et pour l'exécution duquel il pouvait compter sur l'appui de M. Fox $x^{16}$, et probablement sur celui de Lord Durham, peut-être ne le pourrait-il pas, car son influence et sa

14. Il y avait dix ans et plus que nos représentants réclamaient inutilement cette mesure au Parlement.

15. M. l'ambassadeur de France se rendait-il bien compte, en donnant ces sages conseils, de l'état de surexcitation des esprits au Canada tout entier, du fait que la tête de Papineau était mise à prix par le Gouvernement canadien, et que ses propres partisans lui avaient tourné le dos parce qu'ils désapprouvaient sa tiédeur dans leurs entreprises échevelées? Il ne devait pourtant pas songer qu'un homme peut, à lui seul, endiguer un torrent. - La suite de sa lettre nous en fait l'aveu.

16. Ambassadeur d'Angleterre aux Etats-Unis. 
popularité, auxquelles les évènements de l'année dernière avaient déjà porté une rude atteinte, pourraient bien être tout-à-fait nulles aujourd'hui."

Ce jugement d'un diplomate est sans doute excessif à l'égard de Papineau. LaRoque de Roquebrune, en publiant cette correspondance, apprécie la carrière du chef patriote dans les termes suivants:

"Papineau demeure une grande figure canadienne et son auve, en somme, fut féconde. Il faut oublier le côté tapageur de sa réputation, les utopies qu'il nourrissait pour ne plus se souvenir que de la beauté réelle de sa vie et de la grandeur de son caractère. S'il a eu des illusions elles furent généreuses. Et la plus noble de ses idées est peut-être cette confiance qu'il avait dans l'âme canadienne-française."

Parlant ensuite des Patriotes qui surent redevenir des hommes d'Etat et, de révolutionnaires, se changer en constructeurs; il ajoute:

"Papineau seul ne put s'adapter à la nouvelle période. Il demeura homme de 1837. Son attitude ne manqua pas de grandeur, mais elle fut négative. En somme, il ne savait être que d'opposition et il l'est demeuré jusqu'en sa chagrine et mélancolique vieillesse."

Voici, d'autre part, le parallèle que Fauteux établit entre LaFontaine et Papineau:

“Dans toute notre histoire, il n'y eut peut-être pas d'époque plus sombre que celle où Lafontaine dut à la retraite forcée de Papineau de prendre à son tour la direction des destinées canadiennes. Il était vraiment difficile à un homme d'État de recueillir un héritage plus lourd. Le grand orateur, en promenant au-dessus des foules son verbe enflammé, avait mal mesuréles conséquences de son geste erratique, et il n'avait finalement réussi qu'à détruire. Il s'agissait maintenant de reconstruire. Lafontaine n'await pour les siens qu'un seul moyen de salut, et c'était de se servir des armes qui avaient été forgées contre eux. C'est alors que, de l'Union qu'il pouvait bien déplorer mais qu'il savait ne pouvoir empêcher, il conçut l'idée audacieuse et presque téméraire de tirer la charte inespérée de la liberté populaire: le gouvernement responsable... De tenter une semblable entreprise c'était presque une gageure contre l'impossible, et cependant Lafontaine la gagna.

Il la gagna d'abord à force de persévérance et de tenacité, en concentrant constamment son effort sur le but à atteindre et en ne cédant jamais un seul pouce du terrain conquis. Mais il la gagna surtout par sa merveilleuse habileté stratégique. Ce fils d'une lignée de laboureurs n'avait pas seulement le masque d'un Napoléon, il en avait aussi le cerveau puissant. A l'exemple du grand 
capitaine, il excellait à gagner des batailles que l'on aurait crues d'avance perdues."

Cette conquête de nos libertés constitutionnelles s'est donc accomplie par la puissance de la logique aidée de la stratégie du parlementaire, sans effusion de sang. Mais il ne faut pas oublier ceux qui en ont préparé les voies en attịant, par le sacrifice de leur vie, l'attention des arbitres de nos destinées sur les abus de pouvoir d'une oligarchie arrogante. Instruite par l'expérience du passé, l'Anngleterre ouvrit les yeux sur une situation qui pouvait éventuellement compromettre son prestige, autant que ses intérêts, en lui faisant perdre un autre magnifique domaine dans l'Amérique du Nord; elle se soumit à l'inévitable avec élégance, ainsi qu'elle a fait à maintes reprises depuis lors et qu'elle est encore en voie de faire à l'époque où nous vivons.

Inclinons donc nos fronts avec reconnaissance et respect devant les artisans de notre entité nationale. Que les vaincus de la lutte armée partagent avec les lauréats des combats pacifiques le tribut de notre gratitude. C'est par la conjugaison de leurs efforts que la victoire est devenue possible. Et, dans un siècle où l'on peut discuter froidement d'une "République du Canada en union cordiale avec les nations amies de la paix", sans être accusé du crime de lèse-majesté, exprimons au moins une pensée sympathique à l'adresse des instigateurs de la "République canadienne" de 1838.

Novembre 1948

Victor MoRin

N.B. - Avec cette livraison de mars de la Revue, votre abonnement prend fin pour l'année 1948-1949 - Vous vous ferez, sans doute, un devoir de le renouveler le plus tôt possible. 